\title{
Reconhecimento de Padrões Speckle baseado em Textura para Sensoriamento a Fibra Óptica
}

\author{
Ingrid A. Reis, Evandro O. T. Salles, Camilo A. R. Díaz, Arnaldo G. Leal Júnior, e Anselmo Frizera
}

Resumo-Neste trabalho é apresentado um sistema de classificação, baseado na textura do padrão speckle, para determinação de 5 regiões em que uma fibra óptica é perturbada mecanicamente. É realizado um pré-processamento das imagens adquiridas experimentalmente, utilizando dois diferentes descritores de textura, o Local Binary Pattern (LBP) e a Gray Level Cooccurrence Matrix (GLCM), os quais são empregados em uma Rede Neural Artificial. Os resultados mostram que tanto o LBP quanto a GLCM foram capazes de classificar a localização da deformação, atingindo $99,67 \%$ de acurácia com 250 características e $83,47 \%$ com 16 características, respectivamente.

Palavras-Chave-Extração de características, Análise de Textura, Speckle, LBP, GLCM, Redes Neurais

Abstract-This work presents a classification system, based on the texture of the speckle pattern, to determine 5 different regions in which an optical fiber is mechanically perturbed. A pre-processing of experimentally acquired images is performed using two different texture descriptors, the Local Binary Pattern (LBP) and the Gray Level Co-occurrence Matrix (GLCM), and inserted into an Artificial Neural Network. The results show that both LBP and GLCM were able to classify the location of the deformation, reaching $99.67 \%$ accuracy using 250 features and $83.47 \%$ accuracy using 16 features, respectively.

Keywords-Feature Extraction, Texture Analysis, Speckle, LBP, GLCM, Neural Network

\section{INTRODUÇÃO}

O emprego da instrumentação por sensores baseados em fibra óptica em áreas como a medicina, robótica, processos químicos e industriais tem sido crescente nos últimos anos [1], [2], [3]. A robustez, o tamanho reduzido, a flexibilidade e o baixo custo possibilitaram que as fibras ópticas se tornassem material competitivo aos sensores já existentes no mercado. Paralelamente, diversas pesquisas têm sido desenvolvidas sobre esse tipo de sensoriamento, para a mensuração de ampla gama de parâmetros físicos, como pressão [4], temperatura, tensão, deslocamento [5], deflexão [6] e vibração [7].

Os sensores a fibra óptica podem se caracterizar pela forma com que utilizam o conteúdo das ondas de luz. A primeira categoria aborda as características temporais e estão incluídas nela a maior parte das soluções e pesquisas, dentre elas estão os sensores de grade de Bragg, sensores interferométricos e sensores baseados em intensidade. Já as características espaciais são abordadas pela segunda categoria, referentes aos sensores modulados espacialmente, como é o caso dos

Ingrid A. Reis, Evandro O. T. Salles, Camilo A. R. Díaz, Arnaldo G. Leal Júnior, e Anselmo Frizera, Programa de Pós-Graduação em Engenharia Elétrica, Universidade Federal do Espírito Santo, Vitória-ES, E-mails: ingridandradereis@gmail.com, evandro.salles@ufes.br, c.rodriguez.2016@ieee.org, leal-junior.arnaldo@ieee.org, frizera@ieee.org. sensores de fibra baseados em specklegrama (FSSs - Fiber Optic Specklegram Sensors) [8].

Os FSSs se baseiam na distribuição de intensidade dos padrões de manchas (speckles), também chamados specklegramas, resultantes da interferência entre todos os modos propagados na fibra óptica, quando incidida por uma luz coerente. Tendo em vista que a intensidade, o tamanho e a localização dessas manchas mudam com efeitos externos, o FSS pode ser aplicado, por exemplo, para detecção de pedestres [9] e medição de frequência cardíaca de pacientes e de movimento sem contato [10].

Enquanto abordagens que se baseiam na interferometria [11] e em grades de Bragg [12], por exemplo, necessitam de equipamentos caros e volumosos, o FSS apresenta uma alta confiabilidade aliada a uma alta sensibilidade sem que haja a necessidade de um sistema de interrogação tão complexo [13]. Isso é possível em virtude da viabilidade em realizar a gravação dos padrões speckle com apenas uma câmera e um laser.

Em face ao crescimento do ramo de aprendizado de máquina (machine learning) para processamento de imagens, foram elaboradas aplicações ao FSS que utilizassem Redes Neurais Artificial (RNAs) para, além de melhorar o desempenho do sensor, classificar a sua resposta ou extrair características dela, tendo em vista a dificuldade em representar o padrão speckle em equações simples de forma fechada [14]. Suas vantagens, tais como capacidade adaptativa e habilidade de generalizar dados incompletos e ruidosos [14], possibilitaram, por exemplo, o desenvolvimento de FSS para medição de força [15] e classificação da postura das mãos [16] e da região em que de dada perturbação foi aplicada ao longo de uma fibra óptica [17]. Em [17], também foi testada uma rede neural convolucional (CNN - Convolutional Neural Network), mais complexa e robusta, que, no entanto, apresentou menor acurácia que a rede neural feedforward.

Um desafio atual é determinar que informações devem ser analisadas pelo algoritmo de aprendizado de máquina. O processamento de imagens para extração de características anterior à classificação pode se tornar, assim, uma ferramenta aliada tanto ao aumento de seu desempenho quanto a diminuição da complexidade computacional.

Segundo [18], a maioria das técnicas que combinam características de textura e algoritmos de aprendizado de máquina produzem boa acurácia de classificação para imagens diferentes. Assim, devido ao aspecto granular do padrão speckle, a extração de características baseada na textura da imagem se apresenta como uma abordagem promissora.

O presente trabalho busca avaliar se diferentes specklegra- 
mas podem ser representados por características de texturas, de modo a indicar a que distância da fonte luminosa uma perturbação é aplicada na fibra óptica. Para isto, a eficácia de duas técnicas para extração de características baseadas em textura foi investigada para uma rede neural feedforward.

\section{AnÁlise de TeXturas PARA EXTRAÇÃo DE CARACTERÍSTICAS}

No processo de classificação de um padrão, geralmente é utilizada uma representação mais compacta da imagem, chamada de vetor de características, cujo tamanho é determinado pelo número de atributos ou características, e depende dos atributos da imagem a serem utilizados para a classificação [19]. Normalmente, busca-se estabelecer quais atributos podem destacar melhor as propriedades dos componentes da imagem. As principais características usadas para representar uma imagem, de forma a ajudar no processo de análise, são a forma do objeto, sua cor e textura.

Texturas fornecem informações sobre a distribuição das mudanças de tonalidade de um objeto ao longo do espaço, de forma que torna possível que uma dada superfície tenha um aspecto visual mais rugoso ou suave, por exemplo [20].

Ao buscar descrever uma dada textura de acordo com a estimativa da distribuição espacial local do nível de cinza, são destacados os descritores baseados na abordagem estatística. Dentre eles, estão o Local Binary Pattern (LBP) e a Gray Level Co-occurrence Matrix (GLCM).

\section{A. $L B P$}

Proposto em [21], o LBP é um método que resume a estrutura local de uma imagem de maneira não-paramétrica. Tal característica advém da sua construção: nesta técnica, são avaliados se pixels circundantes são maiores ou não que um pixel central, não importando qual a magnitude dessa diferença. Um aspecto notável do LBP é seu número reduzido de operações, possibilitando um baixo custo computacional, mantendo um alto poder discriminativo [22].

O LBP é calculado para cada pixel, tomando as intensidades do nível de cinza de uma vizinhança $(P, R)$ de um dado pixel $g_{c}$, cujas coordenadas são $x_{c}$ e $y_{c}$ com $P$ pontos de amostragem em uma circunferência de raio $R$ [23]. Matematicamente, o operador LBP pode ser definido da seguinte forma:

$$
\operatorname{LBP}_{P, R}\left(x_{c}, y_{c}\right)=\sum_{n=0}^{P-1} s\left(g_{n}-g_{c}\right) 2^{n},
$$

sendo

$$
s(x)= \begin{cases}1 & \text { se } x \geq 0 \\ 0 & x<0\end{cases}
$$

Após aplicar o LBP para todos os pixels da imagem original, a distribuição de frequência dos valores resultantes é calculada, formando o histograma $H_{L B P}$. Esse histograma pode ser utilizado como a entrada de um classificador baseado em textura.

A fim de obter invariância a rotação, foi proposto em [23] o operador $L B P_{P, R}^{r i u 2}$ (Local Binary Pattern Rotation Invariant Uniform), no qual os ângulos entre o pixel central e a vizinhança são quantizados detalhadamente e é calculado por:

$$
\operatorname{LBP}_{P, R}^{r i u 2}= \begin{cases}\sum_{p=0}^{P-1} s\left(g_{p}-g_{c}\right) & \text { se } U\left(\operatorname{LBP}_{P, R}\right) \leq 2 \\ P+1 & \text { caso contrário, }\end{cases}
$$

em que $U$ é a uniformidade do padrão e é dada por:

$$
\begin{aligned}
U\left(\operatorname{LBP}_{P, R}\right) & =\left|s\left(g_{P-1}-g_{c}\right)-s\left(g_{0}-g_{c}\right)\right| \\
& +\sum_{p=1}^{P-1}\left|s\left(g_{p}-g_{c}\right)-s\left(g_{p-1}-g_{c}\right)\right| .
\end{aligned}
$$

Padrões cujos valores $U$ são menores ou iguais a 2 são considerados uniformes. Eles chegam a representar $90 \%$ dos padrões de textura [23]. Assim, é feito um mapeamento no qual há um rótulo para cada padrão uniforme e atribui-se os não-uniformes a um único rótulo. Dessa forma, para 8 pontos de amostragem $(P=8)$, esse mapeamento considera 58 rótulos possíveis para padrões uniformes e 1 para padrões nãouniformes, totalizando 59 rótulos, quando todos os padrões que se diferem apenas pela rotação são considerados, ou seja, quando a variância a rotação é desejada. Para atingir a invariância a rotação, são mapeadas para apenas 10 rótulos, sendo 9 deles referentes a padrões uniformes.

\section{B. $G L C M$}

A Matriz de Coocorrência de Tons de Cinza é um método estatístico utilizado para descrever texturas, proposto em [24]. Nessa matriz são armazenadas probabilidades de que dois valores de intensidade de cinza estejam envolvidos por uma relação espacial.

Uma GLCM corresponde a uma matriz que não depende do tamanho da imagem, mas apenas dos níveis de cinza na textura. De fato, ela é uma matriz quadrada de dimensão $M_{c}$ x $M_{c}$, em que $M_{c}$ é a maior intensidade do nível de cinza na imagem. Antes de ser calculada, define-se a relação espacial a ser considerada por meio dos parâmetros de distância $d$ e o ângulo $\theta$ entre os pixels, formando um arranjo $\mathrm{S}$ de pixels vizinhos. A partir disso, registra-se a frequência $F$ de transições entre cada par de níveis de cinza na $m$-ésima linha e $n$-ésima coluna da matriz, conforme Equação 5:

$$
F(m, n)=\{((i, j),(k, l)) \in S \mid g(i, j)=m, g(k, l)=n\}
$$

em que $g(x, y)$ representa o nível de cinza no pixel localizado em $(x, y)$ na imagem.

Os descritores de textura são extraídos a partir da matriz completa após a normalização baseada na Equação:

$f(m, n)=\frac{F(m, n)}{\sum_{i=0}^{M_{c}} \sum_{j=0}^{M_{c}} F(i, j)} \quad$ para $m, n=0, \ldots, M_{c}-1$.

Cada elemento $(m, n)$ dessa matriz representa a probabilidade de que o valor de cinza $m$ esteja espacialmente relacionado, conforme $d$ e $\theta$, com o valor de cinza $n$. Dessa forma, é possível afirmar que a GLCM caracteriza uma distribuição conjunta de pares de pixels vizinhos, e é a partir dela que são extraídos os descritores. 
Diversas estatísticas podem ser originadas da matriz de coocorrência. Em [24] são propostas 14 medidas cujos médias e intervalos de cada uma compõem um conjunto de 28 atributos que podem ser utilizados como entradas de um classificador. No entanto, muitos deles possuem pouca capacidade de discriminação tendo em vista as correlações entre si. Neste trabalho utiliza-se Homogeneidade, Contraste, Correlação e Energia, conforme:

$$
\begin{gathered}
\text { Homogeneidade }=\sum_{n=0}^{M_{c}-1} \sum_{n=0}^{M_{c}-1} \frac{f(m, n)}{1+(m-n)^{2}}, \\
\text { Contraste }=\sum_{n=0}^{M_{c}-1} \sum_{n=0}^{M_{c}-1} f(m, n)(m-n)^{2}, \\
\text { Correlação }=\sum_{n=0}^{M_{c}-1} \sum_{n=0}^{M_{c}-1} f(m, n) \frac{\left(m-\mu_{m}\right)\left(n-\mu_{n}\right)}{\sigma_{m} \sigma_{n}}, \\
\text { Energia }=\sum_{n=0}^{M_{c}-1} \sum_{n=0}^{M_{c}-1} f(m, n)^{2},
\end{gathered}
$$

em que $\mu_{m}, \mu_{n}$ e $\sigma_{m}, \sigma_{n}$ denotam a média e o desvio padrão das linhas e das colunas, respectivamente.

A Homogeneidade mede a proximidade da distribuição dos elementos à diagonal da matriz de coocorrência. Variando de 0 a 1 , o valor encontrado para esse atributo representa que, quanto maior for frequência de ocorrência de um determinado nível de cinza, maior será a uniformidade local e mais próxima de 1 é a Homogeneidade.

O Contraste mede as variações locais da intensidade do pixel em relação a sua vizinhança. Uma imagem com contraste igual a zero representa que a imagem possui o mesmo tom de cinza em toda sua extensão.

A Correlação mede, para todos os pixels da imagem, o quão correlacionado ao seu vizinho ele está. A correlação entre dois pixels representa se existe uma relação de dependência entre pixels vizinhos, expressa por uma relação linear. Para uma região da imagem onde há pixels vizinhos que podem ser representados por uma combinação linear entre si, a correlação será de -1 ou 1 , a depender do nível de cinza. Além disso, para regiões uniformes, o desvio padrão será zero e, portanto, a correlação terá valor indefinido.

A Energia, também chamada de segundo momento angular ou uniformidade, mede o nível total da intensidade. Em uma imagem que possui o mesmo tom de cinza em toda sua extensão, a sua energia terá o valor máximo de 1 .

Os descritores de Homogeneidade e Contraste baseados na GLCM foram utilizados em [25] para sensoriamento de deslocamento lateral utilizando o specklegrama, que apresentou resultados melhores que uma abordagem baseada em variação de intensidade.

\section{Metodologia}

A fim de avaliar se o uso da textura do speckle pode ser utilizada para caracterizar a localização de uma perturbação na fibra, foi realizado um experimento. Ele consistiu em gravar vídeos das mudanças no padrão speckle, provocadas por deformações estáticas nos mesmos locais ao longo de uma fibra óptica, realizar o processamento e extração das características com base na textura e realizar treino e teste utilizando uma rede neural. Dessa forma, seria possível classificar em que ponto a perturbação ocorreu.

\section{A. Aquisição do Banco de Imagens}

As imagens utilizadas neste trabalho foram obtidas a partir de um sistema construído para perturbar a fibra tendo em vista registrar o comportamento do speckle para cada uma das perturbações. Para isso, foram aplicadas deformações com um peso de $100 \mathrm{~g}$ em 5 pontos espaçados de $1 \mathrm{~m}$ ao longo de uma fibra óptica plástica (POF - Plastic Optical Fiber) de $6 \mathrm{~m}$ de comprimento. Essa POF caracteriza-se por possuir $980 \mu \mathrm{m}$ de núcleo e a espessura de casca é de $10 \mu \mathrm{m}$. Além da fibra e do peso, o sistema desenvolvido conta com um computador de baixo custo do tipo Raspberry Pi 3 Modelo B+ com módulo de câmera de 5MP, baseada em sensor de imagem CMOS e uma fonte de luz coerente. Para a emissão da luz coerente utilizou-se um diodo laser de $637 \mathrm{~nm}$ de comprimento de onda acoplado a um controlador com intervalo de controle de corrente de 0 a $20 \mathrm{~mA}$.

O sistema foi projetado para gravar 5 minutos de vídeo para cada um dos 5 pontos de perturbação, a uma taxa de 90 quadros por segundo e resolução de 640 por 480 pixels. Todos esses vídeos foram salvos no formato H.264 e depois convertidos para MP4 no Raspberry Pi sendo, posteriormente, transferidos a outro computador para conversão para tons de cinza, realização da extração de características e classificação.

\section{B. Extração de Características utilizando LBP}

O primeiro algoritmo de extração de características aplicado foi o operador LBP, para o qual foi escolhido um raio de vizinhança unitário $(R=1)$ e oito pixels na vizinhança $(P=8)$. Esta escolha baseia-se na conjectura deste trabalho de que o specklegrama possui características de microtextura e, por isso, raios e vizinhanças maiores poderiam não ser capazes de representar com suficiente detalhe a representação do padrão de interferência dos modos. Analisou-se tanto o operador LBP com invariância a rotação quanto sem invariância a rotação, a fim de avaliar o impacto na classificação. Com isso, o número de características varia, sendo 10 para o primeiro caso e 59 para o segundo.

Além disso, também foram analisadas pelo LBP diferentes tamanhos das regiões. Similarmente ao desenvolvido em [26], cada imagem é dividida em pequenos regiões (ou blocos) não sobrepostas do mesmo tamanho. Os histogramas gerados pelo LBP são calculados sobre cada região e a imagem é representada por um único histograma formado pela concatenação dos calculados. Por conta da diminuição no tamanho do bloco, esse particionamento possibilita que seja possível obter maior detalhamento local. Esse ganho se dá ao custo de um número maior de características, tendo em vista que a quantidade de histogramas concatenados para uma mesma imagem é maior.

Para a análise utilizando o operador LBP com invariância a rotação, foram utilizados blocos de 3 diferentes tamanhos: o primeiro com as mesmas dimensões da imagem (640x480), o segundo com metade das dimensões da imagem $(320 \times 240)$ e o terceiro com um quinto das dimensões da imagem (128x96), totalizando assim, 10, 40 e 250 características, respectivamente. Já para a análise sem invariância a rotação foram utilizados 2 diferentes blocos, um com o mesmo tamanho da 
imagem gerando 59 características e outro com metade das suas dimensões, que gera 236 características.

\section{Extração de Características utilizando GLCM}

O cálculo da GLCM para extrair atributos que podem representar o speckle depende da relação de ângulo e distância entre pixels vizinhos na imagem. Neste trabalho, tais pares a serem analisados têm distância de $d=1$ entre si e investigouse tanto com apenas 1 orientação, com ângulo $\theta=0^{\circ}$, quanto com 4 orientações, com ângulos $\theta=0^{\circ}, 45^{\circ}, 90^{\circ}$ e $135^{\circ}$.

Diversas propriedades estatísticas podem ser derivadas da GLCM e 4 delas são utilizadas neste trabalho: homogeneidade, correlação, contraste e energia, constituindo as 4 características extraídas deste método para cada orientação investigada.

\section{Arquitetura da Rede Neural}

Para obtenção dos resultados, os algoritmos de aprendizado de máquina foram implementados computacionalmente em MATLAB e executados em uma máquina com processador Intel Core i5 de $2^{\mathrm{a}}$ geração operando a $3,3 \mathrm{GHz}$ e memória RAM de $8 \mathrm{~GB}$. A arquitetura das redes neurais foi perceptron de 3 camadas com algoritmo de retropropagação, sendo elas de entrada, oculta e de saída. A primeira possui quantidade de neurônios variante de acordo com a dimensão dos dados fornecida pelos extratores de características testados, conforme já relacionado. O número de neurônios da camada oculta foi definido como 10 e a função de ativação foi tangente hiperbólica, hiperparâmetros baseado em trabalhos presentes na literatura [8]. A terceira camada possui função de ativação softmax para 5 neurônios, responsáveis pela classificação em alguma das 5 diferentes localidades em que eram realizadas as perturbações.

Para treinar e testar o algoritmo de classificação usando os diferentes conjuntos de dados, empregou-se o método de validação cruzada $K$-Fold. Neste método, conjunto de amostras (vetores de características) é dividido de forma aleatória em $K$ subconjuntos de tamanhos iguais e destes, 1 subconjunto é utilizado para validação e $K-1$ subconjuntos são utilizados para treinamento. Neste trabalho, o método foi realizado com $K=10$.

A medida de desempenho adotada para o treinamento das redes neurais foi dada pela função de custo calculada a partir da entropia cruzada, o algoritmo de treinamento foi o gradiente conjugado escalonado [27]. Além disso, a taxa de aprendizagem foi de 0,01 com um máximo de 1000 épocas e gradiente mínimo de erro de $10^{-6}$. Para o conjunto de validação, o critério de parada consistiu em 6 iterações consecutivas nas quais houvesse crescimento do erro. Ao final de cada um dos experimentos, foram obtidas as medidas de tempo para treinamento e teste e de acurácia média. A acurácia é dada pela média das acurácias de cada uma das 10 simulações com o método $K$-Fold. Nelas, a taxa de acurácia é dada pela razão entre o número de amostras do subconjunto classificadas corretamente e a quantidade total de amostras no subconjunto.

\section{RESUltados}

A Tabela I apresenta o tempo computacional em segundos para a realização da leitura dos vídeos gravados e da extração das características, com LBP e com GLCM. Todos os vídeos totalizavam 45015 frames de 5 diferentes categorias.

TABELA I

TEMPO PARA LEITURA E EXTRAÇÃO DAS CARACTERÍSTICAS DOS frames.

\begin{tabular}{|c|c|c|c|}
\hline $\begin{array}{c}\text { Operador de } \\
\text { textura }\end{array}$ & $\begin{array}{c}\text { Quantidade de } \\
\text { características }\end{array}$ & $\begin{array}{c}\text { Tempo } \\
\text { total (s) }\end{array}$ & $\begin{array}{c}\text { Tempo } \\
\text { por frame }(\mathrm{ms})\end{array}$ \\
\hline \multirow{4}{*}{ LBP } & 10 & $1.439,38$ & 31,98 \\
\cline { 2 - 4 } & 40 & $1.387,87$ & 30,83 \\
\cline { 2 - 4 } & 59 & $1.415,17$ & 31,44 \\
\cline { 2 - 4 } & 236 & $1.460,71$ & 32,45 \\
\hline \multirow{2}{*}{ GLCM } & 250 & $1.457,84$ & 32,39 \\
\cline { 2 - 4 } & 4 & $1.293,15$ & 28,73 \\
\hline
\end{tabular}

Observa-se que o tempo para extração utilizando o LBP não variou significativamente nem com diferentes quantidades de características nem com invariância a rotação, levando em torno de 32 milissegundos para cada frame. Já para a extração com GLCM, o tempo obtido por 4 características de um frame foi menor, com média de 29 milissegundos por imagem. Para 16 características, esse tempo foi 3,2 vezes maior, sendo necessário 69,25 minutos para a leitura e extração das características de todos os frames.

Dessa forma, foram salvos 7 diferentes datasets para treinamento e teste da rede neural, 5 deles referentes ao LBP e 2 deles, ao GLCM. Para cada um, foram analisadas as métricas de acurácia e tempo de execução do algoritmo de classificação, compreendendo treino e teste com validação cruzada. A Fig. 1 apresenta os gráficos correspondentes a estes resultados.

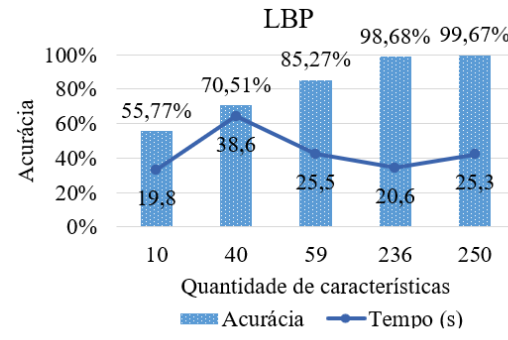

(a)

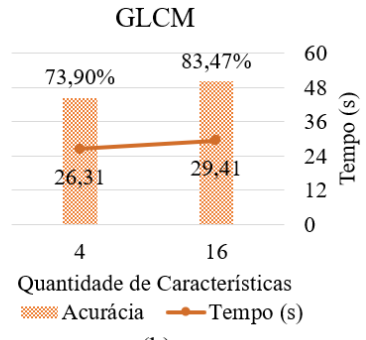

(b)
Fig. 1. Acurácia e tempo para diferentes quantidades de características extraídas com a) LBP e b) GLCM (os eixos de Acurácia e Tempo valem para ambos os gráficos).

Ao avaliar o desempenho da rede neural para classificar um dataset obtido por meio do descritor LBP, nota-se que quanto maior o número de características extraídas, maior é a taxa de acurácia, chegando a 99,67\%. Isso denota que, a depender da quantidade de atributos analisados, a textura do padrão speckle consegue ser bem descrita pelo LBP.

Importante notar que dentre os 3 maiores valores de acurácia, o segundo, dado pelo dataset com 236 características, obtido com operador LBP variante a rotação, apresentou menor tempo. Isso pode indicar que as informações rotacionais do padrão speckle são diferenciais na rapidez da classificação pela rede neural, sem penalizar significativamente a acurácia. 
O operador GLCM apresentou resultados de acurácia relevantes, tendo em vista a pequena quantidade de atributos (4 ou 16) para realizar a classificação. O tempo médio para treinamento e teste da rede neural foi 27,86 segundos.

Importante notar que o descritor GLCM com 16 características apresentou acurácia ligeiramente inferior ao descritor LBP com 59 características, uma diferença de 1,79\%. Além disso, amostras com apenas 4 características extraídas pelo GLCM possibilitaram que a rede neural obtivesse uma acurácia 3,39\% maior em relação às amostras com 40 características extraídas pelo LBP. Tais resultados demonstram a capacidade do GLCM em representar o padrão speckle.

\section{CONCLUSÃO}

Este trabalho se propõe a investigar o uso de descritores de características baseados em textura para classificação de deformações mecânicas realizadas sobre um sensor de fibra óptica baseado em specklegrama. Dessa forma, conjecturouse que cada specklegrama poderia ser tratado como uma textura. Nesse caso, decidiu-se utilizar os operadores LBP e GLCM como descritores de textura e empregou-se uma rede neural rasa feedforward multicamadas para classificar o padrão de texturas e, consequentemente, a localização em que a perturbação é aplicada à fibra.

Os resultados mostram que tanto o LBP quanto o GLCM apresentam boa acurácia na classificação de 5 diferentes classes de região da perturbação, sendo o primeiro descritor o que obteve maior acurácia, de $99,68 \%$ e com menor tempo para extração das características. Também foi avaliado o tempo para treinamento e teste das redes neurais e foi possível verificar um menor tempo quando elas tiveram como entradas as características obtidas pelo LBP variante a rotação, indicando, assim, que o padrão rotacional do padrão speckle é significativo para sua descrição e classificação. Para futuros trabalhos serão avaliados outros tipos de LBP, bem como seu desempenho em outros classificadores além das redes neurais.

\section{AGRADECIMENTOS}

O presente trabalho foi realizado com apoio da Coordenação de Aperfeiçoamento de Pessoal de Nível Superior - Brasil (CAPES) - Código de Financiamento 001, do Conselho Nacional de Desenvolvimento Científico e Tecnológico (CNPq) - Projeto 304049/2019-0 e Projeto 408480/2018-1, Petrobras S.A. - 2017-00702/6 e da Fundação de Amparo a Pesquisa do Espírito Santo (FAPES) no 84336650.

\section{REFERÊNCIAS}

[1] V. Mishra, N. Singh, U. Tiwari, and P. Kapur, "Fiber grating sensors in medicine: Current and emerging applications," Sensors and Actuators, A: Physical, vol. 167, no. 2, pp. 279-290, 2011.

[2] A. G. Leal-Junior, C. Marques, A. Frizera, and M. J. Pontes, "Multi-interface level in oil tanks and applications of optical fiber sensors," Optical Fiber Technology, vol. 40, no. October 2017, pp. 82-92, 2018.

[3] Y. n. Zhang, H. Peng, X. Qian, Y. Zhang, G. An, and Y. Zhao, "Recent advancements in optical fiber hydrogen sensors," Sensors and Actuators, B: Chemical, vol. 244, pp. 393-416, 2017.

[4] V. M. Sperandio, "Um Novo Conceito de Interrogação por Padrões Speckles para Sensores de Pressão aplicados na Indústria de Petróleo e em Áreas Classificadas," Master thesis, Universidade Federal do Espírito Santo, 2015.
[5] M. A. Zawawi, S. O'Keffe, and E. Lewis, "Intensity-modulated fiber optic sensor for health monitoring applications: A comparative review," Sensor Review, vol. 33, no. 1, pp. 57-67, 2013.

[6] S. Razmyar and M. T. Mostafavi, "Deep Learning for Estimating Deflection Direction of a Multimode Fiber from Specklegram," Journal of Lightwave Technology, vol. 39, no. 6, pp. 1850-1857, 2021.

[7] X. Zhan, Y. Sun, F. Xiao, Y. Meng, and J. Zhang, "Research on the Measurement of Heart Rate Based on LD Laser and Multimode Fiber," 2020 5th Optoelectronics Global Conference, OGC 2020, no. 3, pp. 200-203, 2020.

[8] M. Fontana, "Using Machine Learning to Turn Optical Fiber Specklegram Sensor into a Spatially Resolved Sensing System," Master thesis, Università degli Studi di Padova, 2018.

[9] A. Rodriguez-Cuevas, L. Rodriguez-Cobo, M. Lomer, and J. M. LopezHiguera, "Safe and private pedestrian detection by a low-cost fiber optic specklegram," 25th International Conference on Optical Fiber Sensors, vol. 10323, 2017.

[10] A. Rodríguez-Cuevas, E. R. Peña, L. Rodríguez-Cobo, M. Lomer, and J. M. L. Higuera, "Low-cost fiber specklegram sensor for noncontact continuous patient monitoring," Journal of Biomedical Optics, vol. 22, no. 3, p. 037001, 2017.

[11] A. Pospori and D. J. Webb, "Stress Sensitivity Analysis of Optical Fiber Bragg Grating-Based Fabry-Pérot Interferometric Sensors," Journal of Lightwave Technology, vol. 35, no. 13, pp. 2654-2659, 2017.

[12] Y. Haseda et al., "Measurement of pulse wave signals and blood pressure by a plastic optical fiber FBG sensor," Sensors (Switzerland), vol. 19, no. $23,2019$.

[13] A. G. Leal-Junior, "Polymer Optical Fiber Sensors for Healthcare Devices: from Material Analysis to Practical Applications," Ph.D. dissertation, 2018.

[14] H. S. Efendioglu, T. Yildirim, and O. Toker, "Advanced image processing and artificial intelligence based approaches to fiber optic statistical mode sensor design," Smart Sensor Phenomena, Technology, Networks, and Systems 2011, vol. 7982, p. 79820T, 2011.

[15] H. S. Efendioglu, T. Yildirim, O. Toker, and K. Fidanboylu, "Design of intelligent fiber optic statistical mode sensors using novel features and artificial neural networks," INISTA 2012 - International Symposium on INnovations in Intelligent SysTems and Applications, 2012.

[16] E. Fujiwara and C. K. Suzuki, "Optical fiber force myography sensor for identification of hand postures," Journal of Sensors, vol. 2018, 2018

[17] A. Rodríguez-Cuevas et al., "Machine Learning for Turning Optical Fiber Specklegram Sensor into a Spatially-Resolved Sensing System. Proof of Concept," Journal of Lightwave Technology, vol. 36, no. 17, pp. 3733-3738, 2018.

[18] Das, S. and Jena, U. R. Texture classification using combination of LBP and GLRLM features along with KNN and multiclass SVM classification. 2nd International Conference on Communication, Control and Intelligent Systems, CCIS 2016, p. 115-119, 2017.

[19] R. C. Gonzalez and R. E. Woods, Processamento Digital de Imagens, 3rd ed. São Paulo: Pearson Prentice Hall, 2010, vol. 53, no. 9.

[20] W. R. Schwartz, F. R. d. Siqueira, and H. Pedrini, "Evaluation of feature descriptors for texture classification," Journal of Electronic Imaging, vol. 21, no. 2, p. 023016, 2012.

[21] T. Ojala, M. Pietikäinen, and D. Harwood, "A comparative study of texture measures with classification based on feature distributions," Pattern Recognition, vol. 29, no. 1, pp. 51-59, 1996.

[22] S. Marcel, Y. Rodriguez, and G. Heusch, "On the Recent Use of Local Binary Patterns for Face Authentication," International Journal on Image and Video Processing Special Issue on Facial Image Processing, pp. 1-9, 2007.

[23] T. Ojala, M. Pietikäinen, and T. Mäenpää, "Multiresolution gray-scale and rotation invariant texture classification with local binary patterns," IEEE Transactions on Pattern Analysis and Machine Intelligence, vol. 24, no. 7, pp. 971-987, 2002.

[24] R. M. Haralick, K. Shanmugan, and I. Dinstein, "Textural Features for Image Classification," IEEE Transactions on Systems, Man, and Cybernetics, vol. SMC-3, no. 3, pp. 610-621, 1973.

[25] Y. Liu, Q. Qin, H. Liu, Z. Tan, and M. Wang, "Investigation of an image processing method of step-index multimode fiber specklegram and its application on lateral displacement sensing," Optical Fiber Technology, vol. 46, no. June, pp. 48-53, 2018.

[26] L. Chen, Y. H. Wang, Y. D. Wang, and D. Huang, "Face recognition with statistical local binary patterns," Proceedings of the 2009 International Conference on Machine Learning and Cybernetics, vol. 4, no. February, pp. 2433-2439, 2009.

[27] Møller, M. F. A scaled conjugate gradient algorithm for fast supervised learning. Neural Networks, v. 6, n. 4, p. 525-533, 1993. 